AperTO - Archivio Istituzionale Open Access dell'Università di Torino

\title{
A Road Map Toward a Globally Harmonized Approach for Occupational Health Surveillance and Epidemiology in Nanomaterial Workers.
}

\section{This is the author's manuscript}

Original Citation:

Availability:

This version is available http://hdl.handle.net/2318/1623134

since 2018-01-13T08:38:09Z

Published version:

DOI:10.1097/JOM.0b013e31826e27f1

Terms of use:

Open Access

Anyone can freely access the full text of works made available as "Open Access". Works made available under a Creative Commons license can be used according to the terms and conditions of said license. Use of all other works requires consent of the right holder (author or publisher) if not exempted from copyright protection by the applicable law. 


\title{
A Road Map Toward a Globally Harmonized Approach for Occupational Health Surveillance and Epidemiology in Nanomaterial Workers
}

\author{
Michael Riediker, Dr.sc.nat., Mary K. Schubauer-Berigan, PhD, Derk H. Brouwer, PhD, Inge Nelissen, PhD, \\ Gudrun Koppen, PhD, Evelien Frijns, MSc, Katherine A. Clark, DrPH, Juergen Hoeck, PhD, \\ Saou-Hsing Liou, MD, PhD, Sweet Far Ho, MBBS, MSc, Enrico Bergamaschi, MD, PhD, \\ and Rosemary Gibson, DPhil
}

\begin{abstract}
Objective: Few epidemiological studies have addressed the health of workers exposed to novel manufactured nanomaterials. The small current workforce will necessitate pooling international cohorts. Method: A road map was defined for a globally harmonized framework for the careful choice of materials, exposure characterization, identification of study populations, definition of health endpoints, evaluation of appropriateness of study designs, data collection and analysis, and interpretation of the results. Results: We propose a road map to reach global consensus on these issues. The proposed strategy should ensure that the costs of action are not disproportionate to the potential benefits and that the approach is pragmatic and practical. Conclusions: We should aim to go beyond the collection of health complaints, illness statistics, or even counts of deaths; the manifestation of such clear endpoints would indicate a failure of preventive measures.
\end{abstract}

\section{WHY HARMONIZE WORLDWIDE?}

Nanomaterials, because of their novel physicochemical properties, are making their way into businesses and products. After a phase of "nano-hype" with few concrete nanomaterial applications, development of real-life products and processes including nanomaterials is now progressing steadily. Already today, about $1 \%$ of all companies are involved with nanomaterial production or development, ${ }^{1,2}$ although each of them has only a few workers actually dealing with nanomaterials. Nevertheless, with the growing number of existing and novel nanomaterials, their applications, and their incorporation in products, an increasing number of workers are expected to

From the IST Institut universitaire romand de Santé au Travail [Institute for Work and Health] (Drs Riediker and Clark), Université de Lausanne + Genève, Lausanne, Switzerland; National Institute for Occupational Safety and Health (Dr Schubauer-Berigan), Cincinnati, Ohio; TNO (Dr Brouwer), Research group Quality \& Safety, Zeist, the Netherlands; VITO NV Flemish Institute for Technological Research (Drs Nelissen, and Koppen and Ms Frijns), Mol, Belgium; TEMAS AG (Dr Hoeck), Arbon, Switzerland; National Health Research Institutes (Dr Liou), Zhunan Town, Miaoli County, Taiwan; Occupational Safety and Health Division (Dr Ho), Ministry of Manpower, Singapore; Laboratory of Industrial Toxicology (Dr Bergamaschi), University of Parma Medical School, Parma, Italy; and Health \& Safety Laboratory (Dr Gibson), Buxton, the United Kingdom.

This work was funded by grant NMP4-CA-2008-218539 from the European Commission to NanoImpactNet--the European network on the health and environmental impact of nanomaterials, and the contributing authors' institutions

The contents including any opinions, findings, and conclusions in this article are those of the authors and do not necessarily represent or reflect the opinion, policy, or the views of the National Institute for Occupational Safety and Health, the Institute for Work and Health, the Dutch TNO Quality of Life, the Flemish Institute for Technological Research (VITO NV), TEMAS AG, the Taiwanese National Health Research Institutes, the Singapore Ministry of Manpower, the University of Parma, or the UK's Health and Safety Executive.

Address correspondence to: Michael Riediker, Dr.sc.nat., Institute for Work and Health, Bugnon 21, CH-1011 Lausanne, Switzerland (michael.riediker@hospvd.ch).

Copyright (C) 2012 by American College of Occupational and Environmental Medicine

DOI: $10.1097 / J O M .0 b 013 \mathrm{e} 31826 \mathrm{e} 27 \mathrm{fl}$ become exposed to these materials throughout the products' life cycles. In anticipation of this growing market, and in response to debates about the potential health risks of nanomaterials, research is being carried out worldwide in an effort to ensure that there are no adverse health effects from working with nanomaterials. ${ }^{3}$ The early identification of potential health and safety issues indeed allows us to redirect investments for safer future steps in technology development.

Most current research projects in this field focus on exposure measurement techniques and toxicological research that identifies mechanisms of effect and no adverse effect levels in test systems. ${ }^{4}$ Nevertheless, occupational health studies of exposed nanomaterial workers are needed to confirm whether the derived levels are safe and whether the applied safety and preventive health measures are effective. Epidemiological studies of the health of workers producing and using classic carbon black and amorphous silica nanomaterials were carried out in the late 1980s and mid-1990s. ${ }^{5,6}$ Nevertheless, the workforce in individual countries and companies ${ }^{1,7}$ is still small, and there is a large diversity of nanomaterials, which poses challenges for the recruitment of sufficiently large cohorts. This leads to the necessity for pooling cohorts internationally for consideration of novel nanomaterials. The implementation of such studies is currently hampered by (1) few standardized, accurate, and reliable approaches for estimating exposure; (2) large variability in nanomaterial metrics and coexposures; (3) lack of information about health effects and biomarkers; (4) lack of large production forces in this emerging industry, rather a considerable proportion in research and development; (5) questionable statistical power related to currently small workforce sizes and short latency for disease occurrence; and (6) a changing regulatory framework without harmonized registration systems for workers used in these industries. ${ }^{8}$

Numerous different approaches could be adopted for monitoring the health effects of nanomaterials, including occupational health reporting schemes, health surveillance, health risk appraisal surveys, and self-reporting questionnaires. ${ }^{3,9,10}$ Acceptability of different approaches will be strongly influenced by the social contexts and regulatory backgrounds in different countries. These determine where responsibilities lie and who ensures that the required steps in both exposure assessment and health effect monitoring are implemented.

In this report, the most pressing needs in terms of global harmonization were identified as follows:

1. Outline the range of information necessary for epidemiological studies of nanomaterial workers.

2. Evaluate exposure data and models useful for pooled exposure assessments and provide task-based exposure profiles for specific nanomaterials.

3. Link different worker activities and task profiles to job titles, job descriptions, and industries on the one hand and to production processes on the other. 
4. Evaluate health effects and biomarkers for use in future occupational health reporting schemes and epidemiological studies on the basis of knowledge derived from toxicology studies of the subject nanomaterial and inference from epidemiological studies of other nanomaterials.

5. Define the requirements for epidemiological studies and identify suitable cohorts.

6. Provide recommendations for small and medium enterprises and associated organizations regarding early assessment and management of possible risks for nanomaterial workers and the setup of exposure and health effect registries.

Consequently, the rationale for creating this is that before embarking on any large-scale, human health-monitoring studies, it is of paramount importance to determine their feasibility and whether they will ensure useful, reliable results. Basic preparatory work is thus needed to evaluate and analyze existing knowledge, data, and practices for exposure and health effect assessment.

\section{THE ROAD MAP DEVELOPMENT PROCEDURE}

This road map is a product of expert discussions that started in 2008 with the definition of the NanoImpactNet project plan (FP7Grant 218539, www.nanoimpactnet.eu). In 2009, during a NanoImpactNet workshop in Lausanne, Switzerland, participants were asked to answer a series of questions (Table 1) related to the design of occupational health surveillance studies for both short- and long-term monitoring of the health of workers exposed to or handing nanomaterials.

The insight gained from the workshop was condensed into a report, ${ }^{11}$ which was shared with the audience of a meeting organized by the National Institute for Occupational Safety and Health (NIOSH) in Keystone, Colorado, in 2010. ${ }^{12}$ Realizing the potential need for worldwide harmonization, a global group of World Health Organization (WHO) Collaborating Centres and other partners was formed to extend that report's ideas to create this road map. The road map presented here also will serve as a guide for nanomaterialrelated activities of the coauthoring researchers when preparing their contributions to the implementation of the WHO Action Plan 2012 2017.

\section{THE ROAD MAP}

The present road map proposes a strategy to gain global acceptability by many different actors for occupational health studies in the field and to harmonize the collection and storage of data to

TABLE 1. Initial Questions About Issues That a Workers' Health Surveillance Program Needs to Take Into Account

1. Assessing and recording exposure

a. How to gather qualitative and quantitative information on exposure (nature of worker tasks, areas of workplaces, materials being handled, control measures in place, field studies, etc)

b. How to record and access this information

2. Assessing and monitoring health or health surveillance

a. How to define a harmonized approach for data recording and access

b. How to identify "effects" to be monitored, preferably early markers of effect or indicators of biological response as opposed to final outcome (disease and death)

3. Information dissemination

a. How to raise awareness among relevant health care professionals

b. How to disseminate the results of the monitoring to workers and management

c. How to provide support to policymakers and other decision takers yield maximum benefits for all the parties involved. It covers three main domains: exposure, health, and framework conditions related to risk management and study design (Fig. 1).

Global agreement is needed to outline the scope of information that should be collected for epidemiological studies in nanomaterial workers to address the following issues:

- Exposure data measurements and models need to be evaluated and validated so that they are linkable to worker activities, task profiles, job titles, job descriptions, and industries on the one hand and to production processes on the other.

- Potential health concerns need to be identified and biomarkers of effects need to be assessed for detecting short- and long-term effects.

- To facilitate global harmonization, not only differences in risk assessment and management but also data protection philosophies are important while designing epidemiological studies, identifying suitable cohorts, and setting up exposure and health effect registries.

Feedback loops are needed in each domain of activity. Furthermore, the groups working on harmonizing the exposure, health, and risk management and study design domains need to be tightly linked, and considerable cross talk will be needed to ensure a meaningful and efficient harmonization of methods and approaches.

The road map focuses on occupational health studies of the nanomaterial workforce. Nevertheless, data collected from such a harmonized scheme also will be helpful to identify adequate risk management strategies and to evaluate whether existing protective measures are efficient. Thus, the data will be useful to industries using or producing nanomaterials, safety and health experts consulting for these companies, trade unions and politicians who need to respond to concerned constituents, and governmental and international bodies dealing with occupational health and safety issues surrounding nanomaterials.

\section{Exposure to Nanomaterials}

Analysis and recording of exposure to nanoparticles are an important prerequisite for the assessment of their health effects in workers. Nevertheless, in most instances, this still represents one of the weakest stages in the risk management process.

A key requirement for all studies is good-quality data for exposed workers. Measurements need to be feasible and based on direct factual observations, taking into account the use of protective equipment and ventilation of facilities and processes that may limit exposures. Ideally, these exposure studies would be carried out by an occupational hygienist who is able to link data to activities and work processes, but in reality, this may not always be possible. A good nanomaterial exposure assessment should follow these steps:

1. Quantitative and qualitative assessment of potential exposures, including recording the number of companies, as well as, for example, workers handling nanomaterials, quantities of materials used, handling procedures, and whether nanoparticles are dispersed or in powder form during handling.

2. Identification of potential sources of nanoparticle emission and exposure, noting that "emission sources" are different from "exposure sources"; that is, depending on the risk management measures in place, working with a source of nanoparticles does not necessarily correlate with worker exposure to the engineered nanoparticle of interest. This process should include an awareness of work practices.

3. Agreement on and measurement of possible exposure parameters according to a harmonized protocol for all sites. This includes particle metrics and copollutants and contextual information such as ventilation parameters, room size, protection strategies in use, and duration of tasks. 


\section{Roadmap Elements}

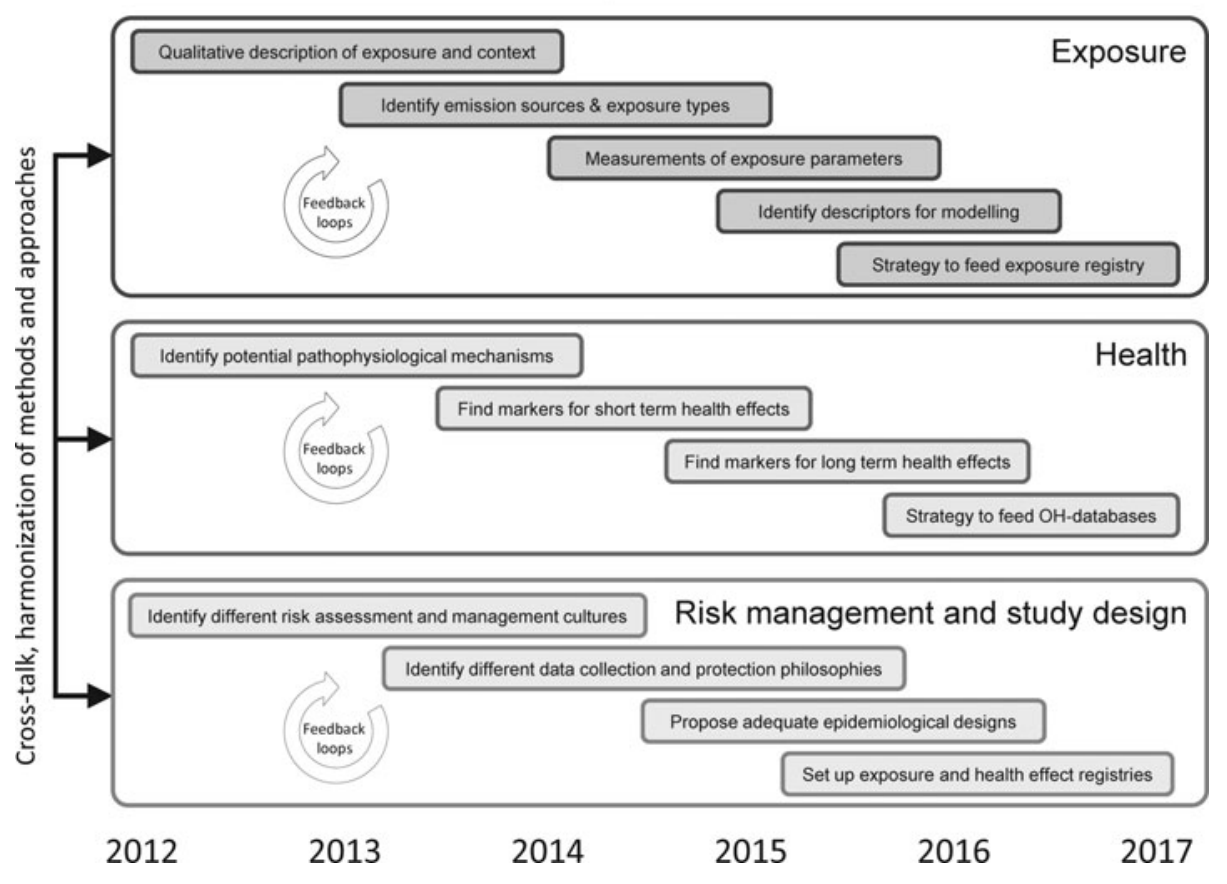

FIGURE 1. Proposed key road-map elements grouped into three action domains with relative timing. Considerable cross talk and harmonization efforts between these domains are required to create a global framework for occupational health $(\mathrm{OH})$ studies. For several elements, nanomaterials pose special challenges related to their complexity and the associated uncertainties.
4. Identification of descriptors for job titles, activities, processes, and industry sectors to build a multidimensional job-activity exposure matrix for the estimation of personal exposure with statistical models.

5. Incorporation of the results into industrial, national, or international exposure registries.

Before exposure registries can start receiving data, a consensus needs to be reached on the following: the nature and minimal quantity of exposure data and contextual information required; how the data should be collected and managed; where it should be stored (eg, in exposure registries, which may need to be country-specific); and who is allowed access and under what conditions. ${ }^{13}$ In addition, cross talk with health care specialists and epidemiologists is necessary to ensure that the parameters allow for the examination of potential links between exposure and effect.

\section{Qualitative Assessment of Exposure}

Exposure to nanomaterials is plausible at all stages of their life cycle, from formulation and production to application in products, use, and disposal. The levels and duration of exposure, as well as the number of individuals affected, will vary at each stage. ${ }^{14}$ Ideally, studies would be based on sufficiently large populations that are exposed to specific, relevant, well-characterized nanomaterials at different levels and for different durations. Nevertheless, this is a real challenge and not easily achievable in today's nanotechnology industries because of a range of complex issues, including costs, identification of workers handling nanomaterials, isolation of effects from specific manufactured nanomaterials versus those from other nanomaterials or chemicals involved in the process, and the fact that identified "high" exposures may lead to immediate corrective actions under the current precautionary stance being recommended by regulators and many other stakeholders. ${ }^{15}$

In reality, although the nanotechnology industries are growing, the number of nanomaterial workers is still quite small, the materials handled are heterogeneous, and the exposures are diverse and continually changing. Information to identify where workers may be exposed can be derived from investigations of the types of materials companies are using, the types of workplaces they have, and protective measures in place, ${ }^{16,17}$ as well as larger, representative surveys to identify the sectors of industry that are using nanomaterials and that can provide information about the quantities involved. ${ }^{1}$ Currently, research facilities are one of the places where many people seem to be handling novel nanomaterials. ${ }^{18,19}$ Nevertheless, universities may not be ideal places for initial monitoring studies because of highly varied work activities and the turnover of personnel. Manufacturing sites with fewer short-term changes may be preferable - there is potential for different processes to give rise to different potential exposures (high vs low) and the best practice to minimize exposure to chemicals in general is already in place and a culture of safety exists. Exposure levels also will be industry-specific. An initial pragmatic solution may be to build on existing field studies, such as those being conducted by national occupational safety and health institutes (eg, the US NIOSH) ${ }^{7}$ or by larger research projects (eg, European Union Framework 7 studies investigating exposure and protection strategies in the workplace, of which an overview is given in the NanoSafety Cluster Compenidum ${ }^{4}$ ).

\section{Agreement on Parameters and Measurement of Exposure}

There is an ongoing debate about which nanomaterial exposure parameters should be recorded..$^{20}$ Good-quality data are required but it is not feasible to take large amounts of measurement equipment into busy workplaces on a routine basis; measurements need to be ongoing and practical. Furthermore, few validated data on which physicochemical characteristics make nanomaterials hazardous or dangerous are available. As this debate continues, pragmatic approaches to exposure measurements are needed. For research purposes, a detailed analysis of a wide range of parameters, including mass, particle number, surface charge, surface reactivity, chemical composition, and characterization by electron microscopy, may be required..$^{21,22}$ Nevertheless, in many other instances (eg, for checking the efficiency of protective measures), measurement of mass, number, surface, or charge of particles may be sufficient, regardless of the size distribution of the nanomaterials. Ideally, any strategy should rely on practical, personal, and real-time monitoring of exposure to 
nanoparticles in areas where exposure has occurred or is likely to occur at high concentrations. The best measurement methods are those that are specific to or highly correlated with the manufactured nanomaterial of interest.

Around the world, leading research groups are using slightly different instruments and methods and thus measuring different aspects of exposure to particles in the ultrafine or nanoscale range. ${ }^{23} \mathrm{Al}-$ though the methodological details differ slightly (eg, as demonstrated by Brouwer ${ }^{20,22}$ ), approaches such as those followed in nanoparticle emission assessment technique (NEAT) ${ }^{24,25}$ and NANOSH ${ }^{22}$ can be integrated to produce a standardized protocol for general nanoscale materials, but they may not indicate exposure to specific nanomaterials. More investigation is needed for these generalized emissionbased approaches to be shown to correlate with more traditional, filter-based industrial hygiene measurements for specific engineered nanomaterials. ${ }^{26,27}$

A series of workshops has been initiated to foster the process of harmonization and the integration of strategies for exposure measurement, analysis, and storage of data (eg, by NanoImpactNet and TNO in Europe and by NIOSH in the United States). Supplementing these measurement approaches, exposure also can be assessed using mathematical models involving key elements, such as the air dispersion characteristics, the manufactured nanoparticle emission rate, and the worker's distance from the emission source. Mechanistic models to estimate exposure to nanoparticles are yet to be developed; however, concepts and tools for such models have been proposed $^{28}$ and can be used to structure the exposure assessment. For some specific activities, that is, powder handling, a more detailed model has been developed. ${ }^{29}$ It is likely that in the near future, further detailed models will be proposed although their calibration will remain a challenge. Currently, some initiatives are being carried out to harmonize strategies to analyze and report measurement data to facilitate future pooling and storage of data. ${ }^{23}$ Such retrievable databases will play an important role in the process of model calibration and validation.

\section{Job-Activity Exposure Models}

For the use of exposure models in epidemiological studies, it is essential that the models are activity- or task-based and provide exposure distributions to account for within- and between-individual variation of exposure. Activity- or task-based models can then be used as building blocks for multidimensional job-activity exposure matrices for estimating personal exposure. An important condition for their use is that the description of tasks and activities is unambiguous. The same holds for the description of other input parameters and contextual information. Another challenge will be to create a comprehensive overview of job titles and activities related to exposure scenarios involving varying levels of exposure. Recent studies suggest that the types of industries where manufactured nanoparticles are used and the types of exposure scenarios vary considerably. ${ }^{1,30}$ Because the formation of cohorts for an epidemiological study may involve combining workers from different companies and possibly different countries, it is essential that job-activity exposure linkages are consistent among populations so that they can be included in pooled epidemiological studies.

An important problem, however, is measuring small numbers of manufactured nanoparticles against a significant background of ambient ultrafine particles, where currently only costly off-site analyses allow distinction of particle types. ${ }^{21}$ Contextual information about work processes such as kinetic, thermal, and other energies involved, ventilation systems, and protective equipment can help in modeling the contributions of task-specific exposure sources and existing hygiene measures. Here, a set of contextual information may replace such measurements for the purpose of large epidemiological studies in which detailed particle assessments at every workplace would not be feasible.

\section{Incorporation into Exposure Registries}

For either short- or longer-term assessment of workers' health, good-quality exposure data are required from workplaces where nanomaterials are routinely used. Thus, studies and exposure characterization campaigns should be promoted, and the information gathered then needs to be shared broadly and made available to researchers investigating occupational health. Companies should be encouraged to keep records of work activities that are as detailed as feasible. Awareness of the need to collect exposure data and establish exposure registries should be raised as widely as possible and at a high level in businesses, with senior managers, safety officers, and occupational health professionals, potentially via continuing professional development and other continuous education programs and professional societies. ${ }^{3}$ These results should then be incorporated into industrial, national, or international exposure registries. An approach to creating such registries is discussed later.

\section{Occupational Health Effect Assessment}

Workers' health should be monitored to ensure that their occupational interaction with nanomaterials does not result in any temporary or permanent harm. To monitor health effects, it is necessary to identify potential biomarkers and assess their relationship with exposure, differentiating between subclinical biomarker effects and health endpoints. Diversity of particle types is a major challenge. Effect markers may provide a useful strategy to address this issue. One proposed approach to addressing the diversity of particle types is to de-emphasize exposure assessment in favor of grouping materials that produce similar pathways to disease. ${ }^{8}$

The following steps should be taken for collecting data for (large-scale) occupational health studies:

1. Identify the pathophysiological mechanisms potentially involved. Identification can be inspired from the mode of action of traditional particles and by deducing the likely consequences of novel physicochemical properties and nano-bio interactions.

2. Once potential pathophysiological mechanisms are identified, it must be determined which bodily responses and diseases might be expected and which markers can be used to evaluate and validate their presence. Because potential exposure to manufactured nanomaterials is new, the initial focus should be on short-term effects, that is, effects that can be observed after a relatively short duration of exposure.

3. Some of the long-term effects that need to be assessed will correspond to the accumulation of low-level effects over time, whereas the onset of others will be delayed such that a relatively short duration of exposure leads to manifestations of effects only much later. Markers are needed that are able to detect both types of long-term effects, and early in the disease's progression when corrective actions are still possible.

4. Health data will need to be collected and fed into health surveillance databases. It may be necessary to set up multiple interlinked databases to account for national differences in health systems and legislation.

Before any (global) health database can be built, a consensus needs to be reached about the health endpoints, the markers, and the methods to be included. The steps toward effective occupational health data collection, as described earlier, require feedback loops and regular revision of the parameters needed and which exposure data can be made available.

The implementation of sound risk management practice should not be delayed until long-term health studies are completed. Although long-term effects can be expected and confirmed in research studies only after nanomaterials have been present for some time, there is an immediate need to take precautionary steps to prevent long-term effects. For example, the recognition of the potential for diseases with delayed manifestation such as mesothelioma from 
inhalation of some types of carbon nanotubes ${ }^{31,32}$ implies the need for immediate and strict precautionary measures. Research will have to confirm at a later stage that the recommended measures were indeed sufficient.

In parallel to health effect studies in workers, full assessment of nanomaterial hazards is required. This will require analysis of all the bodily systems potentially involved and represents one of the main toxicological challenges posed by nanomaterials. Much discussion has taken place about whether the current suite of regulatory toxicity tests is suitable for nanoparticles. NanoImpactNet, other European projects, and the WHO Collaborating Centres are emphasizing the development and validation of in vitro approaches to test the health effects of nanoparticles because these reduce the use of experimental animals. Nevertheless, it must be kept in mind that the unique aspects of nanomaterials may influence both the experimental design and outcomes.

\section{Identify Potential Health Concerns and Mechanisms}

When reducing the size of materials down to the nanoscale, physicochemical properties and interactions with biological systems change. There is considerable uncertainty regarding the health consequences of exposure to these materials. This is partly because of not only the lack of toxicological data but also a consequence of the complexity of the nanomaterial-biotic system interaction. ${ }^{33}$ The testing and sponsorship program of the Organisation for Economic Cooperation and Development ${ }^{34}$ lists more than 30 material properties as being potentially interesting for study. Nevertheless, combining the number of physicochemical properties with the number of health endpoints and testing methods results in an almost unsolvable task. Thus, to save time and efficiently use limited resources (not just financial but also workforce and the number of institutions able to do such extensive testing) prioritization is required.

Prioritization can be based on those characteristics believed to be most relevant and on effect mechanisms. The particle characteristics scientists most frequently cite as important to defining the interaction between nanomaterials and biological systems are size, surface properties, biopersistence (or solubility in biological media), and morphology. ${ }^{35}$ Size is a key aspect because small individual particles may translocate through membranes and tissue barriers, allowing them to travel through the body and reach distal target organs such as the brain and the liver. ${ }^{36}$ The aggregation or agglomeration state of nanoparticles may affect their potential for translocation, whereas smaller primary particle size is associated with a larger surface area per unit of mass and thus a greater surface for biological interactions. Other relevant mechanisms via which nanomaterials are believed to cause effects are particles acting as transport vehicles, ${ }^{37-39}$ leading to the generation of toxic substances such as free radicals and oxidative stress, ${ }^{40-42}$ and by the fiber paradigm, according to which long nanotubes are believed to act similar to long asbestos fibers. ${ }^{32,43-45}$

There is already a wealth of information in the epidemiological literature about exposure to "traditional" or "classic" particles and other (nonengineered) materials that fall within the European Commission's definition of nanomaterials. These workplace fibers, traditional nanomaterials, and environmental airborne particulate matter indicate the types of effects that should be considered, although as yet unknown effects should not be excluded. Studies of ambient particles suggest that particle exposure causes oxidative stress and inflammation, resulting in the release of chemical messengers such as cytokines, and vasomotor factors with subsequent generalized inflammation, thrombosis, atherosclerosis, and potentially chronic obstructive lung disease and pulmonary fibrosis if the target organ is the lungs. ${ }^{46-48}$ Studies of workplace fibers suggest that long, biopersistent fibers can lead over time to serious health outcomes such as mesothelioma, and consequently experts and some national authorities recommend precautionary measures similar to those used for asbestos. $^{15,49,50}$
Experience from the occupational health monitoring of metal welders and flame cutters provides the most applicable example of an available data set, although these workers are not exposed to one single nanosized material but to heterogeneous mixtures of highly reactive metal particles (some in the nanoscale size range) and gases. Reviews of the health effects of these fumes have, however, revealed few effects beyond modest decreases in lung function, airway irritation, and pulmonary siderosis, ${ }^{51}$ with effects in the reproductive systems and central nervous system being inconsistent. The International Agency for Research on Cancer has classified welding fumes and gases as category $2 \mathrm{~B}$ : possibly carcinogenic with limited evidence in humans and inadequate evidence in experimental animals.

In contrast to traditional particles, only limited data are available about novel nanomaterials. A group in Taiwan ${ }^{52}$ has shown promising early results for the use of biomarkers of small airway damage and inflammation, as well as biomarkers of injuries to endothelium and sympathetic nerve activation among workers exposed to nanoparticles. Another study reported on seven Chinese workers handling a range of chemicals and nanoparticles who became ill, with subsequent pathological examinations of lung tissue revealing evidence of pulmonary inflammation, fibrosis, and foreign-body granulomas, and nanoparticles were observed in pulmonary epithelial and mesothelial cells. ${ }^{53}$ Nevertheless, poor occupational hygiene and the workers' complex exposures preclude definitive conclusions about the contribution of nanoparticles to the effects observed.

\section{Strategy to Identify Short- and Long-Term Health Effect Markers}

Once potential endpoints or mechanisms of action have been identified, markers of effect need to be identified and validated. Biochemical tests or functional parameters to be assessed should be supported by consistent pathophysiological mechanisms. Attention could be focused on exposure via inhalation and the skin because these routes of exposure are better understood for nonnanoscale chemicals. Pulmonary and cardiovascular diseases (leading to increased morbidity and mortality among vulnerable groups in particular) have been linked to pollution and levels of ultra-fine particles, but their use as health endpoints for workers exposed to manufactured nanomaterials have limitations: they are nonspecific (and certainly not nano-specific), have a high prevalence in the general population, and share multiple nonoccupational risk factors. Thus, information about exposure to these other risk factors would also have to be acquired to allow attribution of effects (and it would be only on the level of worker populations and not individuals). Although these factors make long-term studies more challenging to conduct and interpret, they have been used in studies of many occupational diseases.

A variety of other potential short- or long-term effect parameters have been proposed for a targeted assessment of personnel exposed to nanomaterials. These include heart rate variability, blood-clotting parameters, proinflammatory cytokines, upregulation of adhesion molecules or antioxidant capacity, and biomarkers of pulmonary fibrosis. ${ }^{46,54-56}$ These biomarkers are increasingly used to assess cardiovascular effects of fine particulate matter, quasi-ultrafine (ie, particulate matter $<0.25 \mu \mathrm{m}$ ) aerosols, and primary carbon aerosols derived from traffic-related sources. ${ }^{57-60}$ These biochemical parameters have consistent pathophysiological mechanisms that have been investigated for combustion-derived ultrafine particles and diesel exhaust particles generated in laboratory settings. ${ }^{61}$ Although promising in epidemiological research as putative biomarkers of effect, these parameters are still not assessable for their predictive value of health risk at an individual level; they are not routinely applicable and need to be further validated.

On the basis that the potentially relevant health endpoints that have been tentatively ascribed to manufactured nanoparticles are cardiovascular, pulmonary, and inflammatory effects, possible health monitoring endpoints include the following: 
- Assessment of markers of exposure (eg, presence of chemicals in the blood or the urine; this can readily be done for chemicals such as metals--strictly speaking, not an endpoint).

- Chemical changes in exhaled air or exhaled breath condensate suggested to reflect not only abnormalities of the airway lining fluid and lung inflammation ${ }^{62-64}$ but also potential exposure. ${ }^{65}$

- Local effects: inflammatory changes, short-term respiratory changes, respiratory, eye, or skin irritation, depending on the route of exposure/site of uptake (with special tests to study biopersistent long fibers such as some forms of carbon nanotubes).

- Systemic effects to confirm cardiovascular changes and inflammatory mechanisms: heart-rate variability, platelet aggregation, and other prothrombotic effects, as well as cytokines and differential blood cell counts. ${ }^{54,66}$

- Medical tests for early detection of health effects at a preclinical stage (eg, clinically validated biological markers of cardiovascular, hepatic, renal, hematological, or respiratory dysfunctions).

We do not yet know whether or how such health effects may differ between chemically or structurally different particles at the nanoscale. To date, biomarkers of exposure cannot be adequately developed because of the lack of consistent toxicokinetic studies of nanomaterials, which may be partly related to the enormous variability of surface properties for each type of nanomaterial and even between batches of the same material. Nevertheless, it is likely that traditional biomarkers of exposure (eg, mass quantification in serum or urine) will be more feasible for nanoscale metals than for carbonaceous nanomaterials. At present, it is unknown whether manufactured nanoparticles in general, and carbon nanotubes in particular, can exacerbate preexisting medical conditions or increase the susceptibility to certain diseases.

\section{Occupational Health Databases}

Health data collection should be tightly linked to exposure assessment. At a minimum, health data collection should be accompanied by a general exposure assessment at workplaces with identified risk potential. An occupational health database will need to allow the identification of exposed individuals, worker populations, or both. Ideally, such a database would be held at a centralized data collection site with extended health screening at specific nanomaterial facilities. In addition to this basic data collection, targeted research studies will be needed with detailed exposure assessment and extended health effects monitoring, including mechanistic studies of the effects of nanomaterials. As for exposure, companies should be encouraged to keep records, awareness about the need for data collection should be raised, and the data should be incorporated into industry, national, or international health registries. It would be useful if the databases can be linked to existing databases such as national or international registries of death and cancer.

\section{Harmonized Study Designs and Data Collection Strategies}

The success of epidemiological studies for quantitative risk assessment depends on the quality of available exposure and health response data. To achieve a coherent approach that leads to valid conclusions, data collection needs to be defined in anticipation of future (ideally prospective) studies that will pool and compare different situations worldwide. Over the coming years, the following points need to be addressed:

- Risk assessment and management cultures in different industrial sectors and countries need to be identified.

- Data collection strategies, data protection philosophies, and the associated legal systems of different countries need to be assessed and strategies for dealing with them defined.

- The most suitable epidemiological designs for different purposes need to be identified.
- Exposure and health registries need to be established that can link to health surveillance systems or epidemiological research projects.

It is unlikely that a single, global project on this scale could be set up to follow workers exposed to nanomaterials. Nevertheless, a more modest approach with harmonized exposure and health registries could be established, recording details of workers' activities, available information about exposure levels, the nanomaterials handled, and health condition. Ideally, workers' samples (eg, blood and urine) would be stored for later analysis as more potential biomarkers of effect and exposure become available.

\section{Identify Ethical, Cultural, and Regional Differences}

Any global data collection strategy needs to account for regional differences in existing technologies, exposure protection strategies, safety culture, data protection philosophies, and ethical aspects. ${ }^{67}$ These challenges are superimposed on the challenges and data needs for the conceptual models described earlier. For each of the recognized data-collection needs, potential challenges for collecting (in the sense of being able to obtain) and defining (in the sense of using terminology that is free of sensitive connotations) such data need to be identified. Differences in data protection laws might pose challenges not only to the collection ${ }^{68}$ but also to the pooling of data across frontiers. Usually, data can be used only for a predefined purpose, and subjects' identities must not be revealed. In some countries, individuals can request that their data be withdrawn at any moment during the study, often there is an expiration date relating how long data can be stored, and data may be shared only with researchers located in countries that have at least the same regulation of data protection.

All of these challenges need to be described and addressed in a strategy on how to overcome the differences and gaps when defining the data needed for collection. Thus, information about cultural and regional differences should be an integral part of the recommendations for a globally harmonized data collection strategy. WHO Collaborating Centres and the Organization for Economic Cooperation and Development may have an important role to play in the collection of such data about cultural and regional differences.

\section{Define Epidemiological Designs}

Well-conducted epidemiological studies are an important source of information to risk assessment because they provide directly relevant human data. Epidemiological studies face challenges due to uncertainties in exposure measurements, dose to the target organ, and health effects resulting from the interaction of cells with nanomaterials. Nevertheless, examples exist (eg, radon-exposed miners ${ }^{69}$ and asbestos-exposed textile workers ${ }^{70}$ ) in which prospective cohort studies have produced data that form the basis of quantitative risk assessments.

Currently, the number of workers facing a potential exposure to manufactured nanomaterials is not known. Major challenges in conducting prospective cohort studies of nanomaterial workers include developing a large cohort size and the long periods of time required to draw firm conclusions regarding chronic health effects. In the interim, small-scale studies of 50 to 100 workers could be conducted within the next 5 years to assess biomarkers of exposure or of early effect. ${ }^{9}$

Although there is currently no firm basis for the recommendation of targeted nano-specific occupational health surveillance for most manufactured nanomaterials, this should not be a reason for paralysis or inactivity. There are clear knowledge gaps and achievable recommendations can be made:

1. General health surveillance should target those working with nanomaterials where exposure is likely (eg, processes that are not 
contained), and the systems for recording the processes and types of nanomaterials used by the workers should be improved.

2. Workplaces should apply measures to control exposure, containing particle emissions, and deploying personal protective equipment for workers when appropriate if potential exposure to nanomaterials cannot be excluded. The measure should be recorded, ideally in a standardized reporting format, during occupational health studies.

3. Simple questionnaires could be used for self-reporting symptoms so that focused studies can be undertaken for different types of nanomaterials and industries.

4. Individual cases of ill health in those working with nanomaterials should be scrutinized to assess whether nanomaterials are a likely attributable source of the ill health.

5. Improved sharing of knowledge should be encouraged at national and international levels through occupational health-reporting networks.

6. Simple nanomaterial measurement techniques are required that can be applied under most occupational circumstances without advanced technical knowledge.

7. Use should be made of biological monitoring (where applicable).

Overall, there is a clear need to gather experimental, clinical, and epidemiological data to characterize the relationship between exposure and health outcomes and provide a basis on which to build and explore the effectiveness of preventative measures.

\section{Health Surveillance and Setting Up Registries}

The rapid development of exposure registries will help provide data for the epidemiological studies of the future. ${ }^{3,71}$ Nevertheless, there are currently no validated methods for health monitoring and reporting that are specific to nanoparticles, but existing approaches for nonnanoscale substances could be adapted. In the United Kingdom, occupational health reporting systems have been developed, but they rely on reporting (sometimes self-reporting) of adverse outcomes, and they rarely record information about exposure levels. Furthermore, if effective preventive approaches are adopted in workplaces handling nanomaterials, the number of expected adverse outcomes could be very small or even nonexistent. In France, basic information about workers' health status is already being collected via mandatory occupational health surveillance, and this will be available in the future for retrospective studies. ${ }^{10}$ The approach recommended by $\mathrm{NIOSH}$ in the United States is to consider the hazard and exposure levels of nanomaterials when making a decision about whether to use routine medical surveillance. ${ }^{72}$

Historically, in occupational medicine, the practice of health surveillance has represented the final step in a process based on the integration of both experimental and epidemiological studies that identify the hazards and are supported by the implementation of occupational exposure limits. Medical surveillance requires at least a qualitative risk assessment and can be implemented when a residual risk exists and when the target population has been clearly identified. ${ }^{72}$ One major issue is the correct classification of exposure situations and linking them to the medical records. Currently, validated or even calibrated exposure models are lacking; however, the application of such models for estimating the exposure of individuals will be hampered by the lack of indications of within- or between-individual (worker) variances.

Although there are no specifically validated methods for the risk assessment of nanomaterials in the workplace, the occupational safety community may be underutilizing its existing knowledge of hazard and exposure control of ionizing radiation, biological agents, pharmaceuticals, nuisance dusts, and pollution. ${ }^{73}$ With technology developing so rapidly, it will be necessary to make continual reassessments and reevaluations of the risks to workers' health and safety from nanotechnologies and nanomaterials in particular. It is suggested that existing regulations can address the emerging issues and potential hazards presented by nanoparticles in the workplace, and although some disagree with this view, guidance and codes of conduct have been produced by different organizations (eg, European Commission, UK's Health and Safety Executive, ${ }^{15}$ and $\mathrm{NIOSH}^{74}$ ). Although incomplete, the body of toxicological evidence on manufactured nanomaterials suggests that occupational hygienists and health and safety professionals should recommend precautionary management measures in the workplace, including in research laboratories handling nanoparticles.

Risk management of nanomaterials in the workplace would involve recognition of potential worker exposure and the implementation of measures to reduce or minimize $\mathrm{it}^{75}$ Occupational health surveillance and epidemiological research can support the risk management process not only by assessing whether health effects can be found at levels believed to be safe but also by identifying the risks to workers where the exposure is insufficiently controlled. ${ }^{3}$ Thus, health surveillance and epidemiology are important tools to ensure workers' health. Medical screening constitutes just one part of a complete health and safety management program. National Institute for Occupational Safety and Health has published interim guidance on medical screening for workers handling nanomaterials, ${ }^{72}$ which discusses the value of medical screening for asymptomatic workers. A second NIOSH guidance document ${ }^{50}$ describes the need for medical surveillance for carbon nanotubes, which demonstrate a specific hazard in toxicological studies.

\section{Harmonization of Studies}

To enable the harmonization of studies, preparatory steps such as the establishment of exposure and health effect registries may be needed. Occupational exposure and health registries have been used in public health for more than 50 years and are especially useful when the risks to workers are not well defined. Recently, the Dutch Health Council and the Social and Economic Council of the Netherlands strongly recommended the establishment of an early warning system and exposure registries for nanomaterial workers (http://www.gezondheidsraad.nl/sites/default/ files/Nanotechnologie2.pdf, http://www.gezondheidsraad.nl/sites/ default/files/200818.pdf, http://www.ser.nl/ /media/DB_Adviezen/ 2000_2009/2009/b27741.ashx).

We propose generation of an international framework under which such registries could be developed with a harmonized format and interlinked across Europe and globally. The proposed actions and related objectives are shown in Table 2.

Development of such registries would ideally occur on an international scale to increase the numbers of workers recruited, although national views and regulations about data gathering and security may complicate this. These problems could be avoided by establishing national registries that collaborate internationally.

Such a system for international surveillance can be set up in analogy to initiatives done for assessment of background exposure in the normal population. Indeed, within the frame of the FP7 EU project COPHES and the Life+ supported project DEMOCOPHES, a strategy for harmonized human biomonitoring in $17 \mathrm{EU}$ countries is currently worked out. In parallel with what was done there, also for occupational exposure to nanomaterials, a pilot monitoring program can be worked out to start organizing registries, measurements, or both, starting on a limited scale. The study should be coordinated and steered by a limited group of a few partners who prepare the pilot study. In this context, it would be good to include partners of industry and occupational associations in this steering group. If there are enough resources, other participants can join and should be fully supported by providing all necessary (technical) advice and training.

To obtain wide acceptance of and support for such approaches, communication will be a very important element. This communication needs to reach workers, managers, policymakers, and research 
TABLE 2. Central Issues for Study of Workers' Exposure and Health

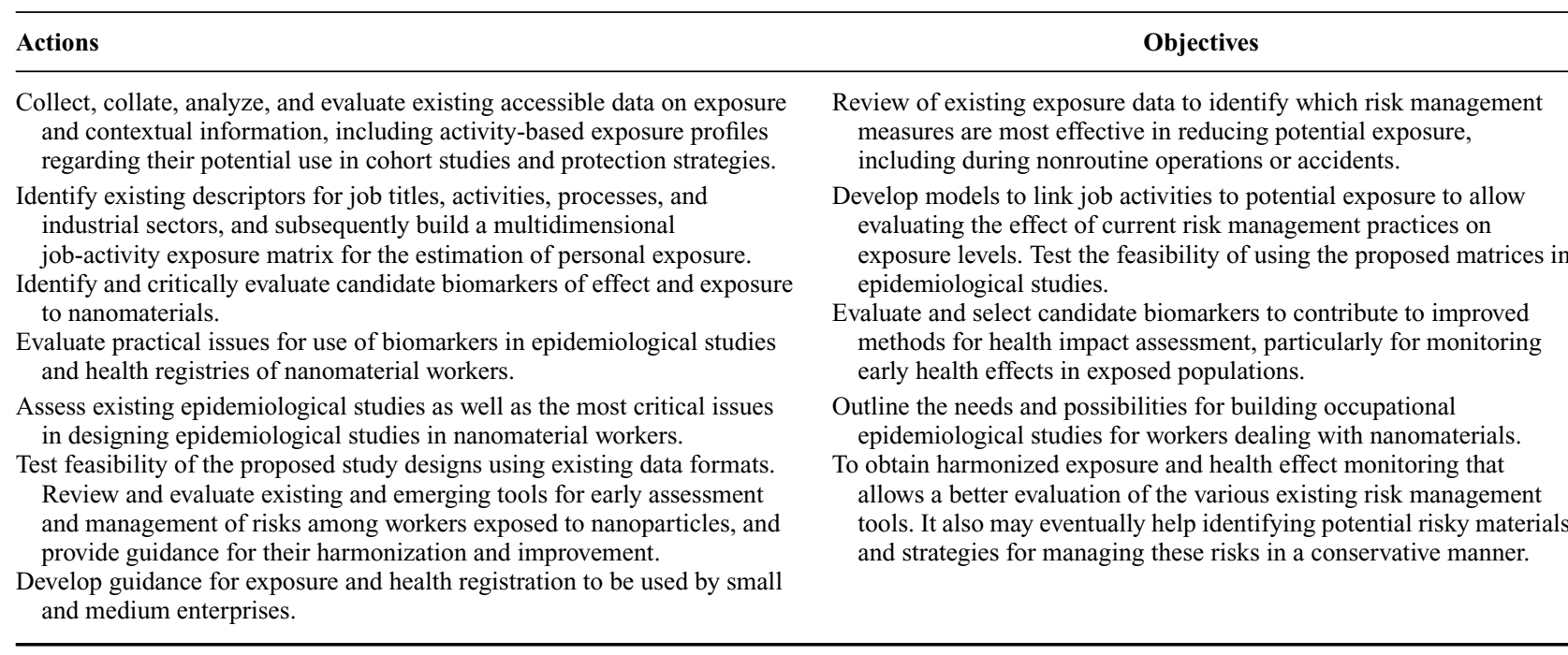

funding agencies. It was recommended that communication about nanomaterial risks should adopt established concepts of risk perception and risk communication strategies. ${ }^{76}$

\section{Check Appropriateness of Screening Strategies}

When hazard data are absent (as with many nanomaterials), the issue is whether it is possible or informative to initiate specific health surveillance for nanotechnology workers. ${ }^{71}$ Unnecessary health surveillance itself can be associated with risks if it leads to incorrect diagnoses, uncertain interpretation, and the perception that work may not be "safe" for workers. Criteria of appropriateness should, therefore, be assessed; that is, any screening strategy for a worker's health should be justified on the basis that (1) finding a medical condition at an earlier stage significantly improves the potential outcome compared with a situation with no screening; (2) especially in the case of chronic diseases, it helps reduce the population's exposure to a level that prevents the development of these early stages of disease; or (3) the screening program is used as part of a wider strategy to ensure effective exposure control.

A range of factors needs to be considered to determine whether implementing health surveillance in the workplace is appropriate. Among the existing criteria are assessment of the burden of suffering (which the precautionary approach aims to prevent), the accuracy and reliability of current test methods, the effectiveness of early detection in the absence of clear correlations to health endpoints from manufactured nanomaterials, and an assessment of the benefits versus any harm resulting from the screening itself. On the basis of these criteria, the known risks associated with some screening methods (eg, chest radiography or computed tomography) would have to be outweighed by the benefits, such as implementation of workplace risk management measures or preventative health interventions, before such a recommendation could be made. Routine screening has to be justified on the basis of a sufficient likelihood of the expected exposure to manufactured nanomaterials causing the condition in question; the absence of any indication of a risk attributable to nanoparticle exposure precludes conclusions on this point at present.

\section{CONCLUSIONS AND RECOMMENDATIONS}

To best understand and control the health and exposure risks of workers dealing with manufactured nanomaterials, studies of groups and cohorts of those workers are necessary. At the moment, the nec- essary framework conditions to conduct such studies are not in place. To provide a coherent approach and make future epidemiological research a reality, a well-defined framework is needed for the careful choice of materials, characterization of exposure, identification of study populations, definition of health endpoints, and evaluation of the appropriateness of study designs. Particularly needed are

- A basis for prioritizing which engineered nanomaterials merit investigation (eg, on the basis of toxicological or inferential studies).

- A consistent, evidence-based set of job titles and task-based exposure profiles for epidemiological studies (informed by actual individual workplace assessments).

- A method for linking industry, company, and job descriptors to exposure and consistent exposure metrics; this will need to provide recommendations on the type and format of data to be collected and how it can be interlinked so that future studies can use the data for developing exposure estimates for large cohorts of workers.

- Criteria for potentially useful biomarkers and (pre)clinical parameters for epidemiological studies about workers in small and medium enterprises and transnational companies. Recommendations on the feasibility of human population studies based on these biomarkers.

- Recommendations on the requirements for harmonized approaches for human biomonitoring and health effect studies tailored to nanomaterial workers.

- Recommendations for harmonization and the improvement of tools for early assessment and management of risks and exposure registration. These should be made available to relevant stakeholders, including small and medium enterprises, along the global value chain.

For either short- or longer-term assessment of worker health, good-quality exposure data are required from workplaces where nanomaterials are routinely used, and methods for collection of exposure data should be tested, validated, and agreed upon internationally with some urgency. Concomitantly, field studies and exposure characterization campaigns should be promoted and information shared broadly. Companies should be encouraged to keep records of work activities that are as detailed as feasible, and, if possible, full use should be made of simple sensors, such as particle counters (recognizing that these are not at all specific for the manufactured nanomaterial of interest). Awareness of potential hazards and the need to collect exposure data and establish exposure registries should be 
raised as widely as possible, potentially at a high level in businesses, with senior managers, safety officers, and occupational health professionals, potentially via continuing professional development and other programs of continuing education.

Although at present no nano-specific screening procedure exists for the medical surveillance of workers potentially exposed to nanoparticles, periodic general medical examinations are to be recommended, preferably on the basis of noninvasive procedures. Increasing general health surveillance for these workers could lead to an earlier recognition of adverse effects and information about which symptoms should be observed in other similarly exposed workers. This might also lead to informative individual index cases being identified.

There is an urgent need for further toxicological evaluation and physicochemical characterization of all the types of nanomaterials currently handled in workplaces, and a determination of the relationship between particle characteristics and health effects so as to facilitate prediction of the effects of new materials, and to identify the most important exposure characteristics to be assessed. Biomarkers of exposure and health effects also are required, and novel, ideally point-of-care, detection techniques need to be explored for assessing those exposures, for example, via determination of particle numbers or proinflammatory cytokines in exhaled breath condensate.

In spite of the advances in establishing a conceptual framework leading to a higher degree of worker protection, epidemiological studies of workers potentially exposed to manufactured nanomaterials will be difficult to conduct for both ethical and practical reasons. These include the heterogeneity of nanomaterials used in occupational settings, the overlap in exposure between combustionor pyrolysis-derived ultrafine particles and manufactured nanoparticles, the lack of standardized exposure metrics, the long time frame required to develop informative exposure histories, and the international cultural differences related to surveillance systems, data recording and storage, and data protection. Further issues include how to distinguish health effects that may arise from exposure to nanomaterials from those due to exposure to other workplace hazards and potential toxicants and the effects of multiple exposures and confounding factors, such as smoking and underlying health conditions.

The proposed road map addresses a joint strategy and the flow of actions needed to achieve these goals. Such a joint strategy will ensure that the costs of action are not disproportionate to the potential benefits and, importantly, that the strategy will be pragmatic and practical. The discussion about the benefits and challenges associated with the different potential approaches for occupational health surveillance and epidemiological studies for workers exposed to nanomaterials has only just begun. Nevertheless, results from such studies are needed for the assessment of nanomaterial workers' risk and for evaluation of exposure controls.

\section{ACKNOWLEDGMENTS}

We thank Markus Berges, IFA (DGUV), St Augustin, Germany, and Rob Aitken, Institute for Occupational Medicine, Edinburgh, United Kingdom, for their review and for the intent to support the implementation of the here proposed road map.

\section{REFERENCES}

1. Schmid K, Danuser B, Riediker M. Nanoparticle usage and protection measures in the manufacturing industry - a representative survey. JOccup Environ Hyg. 2010;7:224-232.

2. Invernizzi N. Nanotechnology between the lab and the shop floor: what are the effects on labor? J Nanopart Res. 2011;13:2244-2268.

3. Schulte PA, Trout DB. Nanomaterials and worker health: medical surveillance, exposure registries, and epidemiologic research. J Occup Environ Med. 2011;53:S3-S7.
4. Riediker M, Katalagarianakis G, eds. Compendium of Projects in the European NanoSafety Cluster - 2012 Edition. Lausanne, Switzerland: Institute for Work and Health; 2012.

5. Merget R, Bauer T, Kupper HU, et al. Health hazards due to the inhalation of amorphous silica. Arch Toxicol. 2002;75:625-634.

6. Gardiner K, van Tongeren M, Harrington M. Respiratory health effects from exposure to carbon black: results of the phase 2 and 3 cross sectional studies in the European carbon black manufacturing industry. Occup Environ Med. 2001;58:496-503.

7. Schubauer-Berigan MK, Dahm MM, Yencken MS. Engineered carbonaceous nanomaterials manufacturers in the United States: workforce size, characteristics, and feasibility of epidemiologic studies. J Occup Environ Med. 2011;53:S62-S67.

8. Schulte PA, Schubauer-Berigan MK, Mayweather C, Geraci CL, Zumwalde R, McKernan JL. Issues in the development of epidemiologic studies of workers exposed to engineered nanoparticles. J Occup Environ Med. 2009;51:323335 .

9. Laney AS, McCauley LA, Schubauer-Berigan MK. Workshop summary: epidemiologic design strategies for studies of nanomaterial workers. J Occup Environ Med. 2011;53:S87-S90.

10. Boutou-Kempf O, Marchand JL, Radauceanu A, Witschger O, Imbernon E; Group Health Risks of Nanotechnologies. Development of a French epidemiological surveillance system of workers producing or handling engineered nanomaterials in the workplace. J Occup Environ Med. 2011;53:S103-S107.

11. Gibson RM, Adisesh A, Bergamaschi E, et al. Strategies for Assessing Occupational Health Effects of Engineered Nanomaterials. NanoImpactNet Report. Lausanne, Switzerland: Institute for Work and Health; 2010.

12. Schulte PA, Trout DB, Hodson LL. Introduction to the JOEM supplement nanomaterials and worker health: medical surveillance, exposure registries, and epidemiologic research. J Occup Environ Med. 2011;53:S1-S2.

13. Schulte PA, Mundt DJ, Nasterlack M, Mulloy KB, Mundt KA. Exposure registries: overview and utility for nanomaterial workers. J Occup Environ Med. 2011;53:S42-S47.

14. Maynard AD, Aitken RJ. Assessing exposure to airborne nanomaterials: current abilities and future requirements. Nanotoxicology. 2007;1:26-41.

15. HSE Books. Risk Management of Carbon Nanotubes. Sudbury, UK: HSE Books; 2009.

16. Schmid K, Riediker M. Use of nanoparticles in Swiss industry: a targeted survey. Environ Sci Technol. 2008;42:2253-2260.

17. Aitken RJ, Chaudhry MQ, Boxall ABA, Hull M. Manufacture and use of nanomaterials: current status in the UK and global trends. Occup Med. 2006;56:300-306.

18. Balas F, Arruebo M, Urrutia J, Santamaria J. Reported nanosafety practices in research laboratories worldwide. Nat Nanotechnol. 2010;5:93-96.

19. Groso A, Petri-Fink A, Magrez A, Riediker M, Meyer T. Management of nanomaterials safety in research environment. Part Fibre Toxicol. 2010;7:40.

20. Brouwer D. Exposure to manufactured nanoparticles in different workplaces. Toxicology. 2010;269:120-127.

21. Kuhlbusch TAJ, Asbach C, Fissan H, Göhler D, Stintz M. Nanoparticle exposure at nanotechnology workplaces: a review. Part Fibre Toxicol. 2011;8:22.

22. Brouwer D, van Duuren-Stuurman B, Berges M, Jankowska E, Bard D, Mark D. From workplace air measurement results toward estimates of exposure? Development of a strategy to assess exposure to manufactured nano-objects. J Nanopart Res. 2009;11:1867-1881.

23. Brouwer D, Berges M, Virji MA, et al. Harmonization of measurement strategies for exposure to manufactured nano-objects: report of a workshop. Ann Occup Hyg. 2012;56:1-9.

24. Methner M, Hodson L, Dames A, Geraci C. Nanoparticle emission assessment technique (NEAT) for the identification and measurement of potential inhalation exposure to engineered nanomaterials_part B: results from 12 field studies. J Occup Environ Hyg. 2009;7:163-176.

25. Methner M, Hodson L, Geraci C. Nanoparticle emission assessment technique (NEAT) for the identification and measurement of potential inhalation exposure to engineered nanomaterials-part A. J Occup Environ Hyg. 2009; 7:127-132.

26. Birch ME, Ku BK, Evans DE, Ruda-Eberenz TA. Exposure and emissions monitoring during carbon nanofiber production - part I: elemental carbon and iron-soot aerosols. Ann Occup Hyg. 2011;55:1016-1036.

27. Dahm MM, Evans DE, Schubauer-Berigan MK, Birch ME, Fernback JE. Occupational exposure assessment in carbon nanotube and nanofiber primary and secondary manufacturers. Ann Occup Hyg. 2012;56:542-556.

28. Schneider T, Brouwer DH, Koponen IK, et al. Conceptual model for assessment of inhalation exposure to manufactured nanoparticles. J Expos Sci Environ Epidemiol. 2011;21:450-463. 
29. Jensen H, Pedersen JH, Jorgensen JE, et al. Determination of size distributions in nanosized powders by TEM, XRD, and SAXS. J Exp Nanosci. 2006;1:355373 .

30. Schmid K, Danuser B, Riediker M. A low number of Swiss companies uses nanoparticles. Swiss Med Wkly. 2009;139:18S.

31. Donaldson K, Murphy F, Duffin R, Poland C. Asbestos, carbon nanotubes and the pleural mesothelium: a review of the hypothesis regarding the role of long fibre retention in the parietal pleura, inflammation and mesothelioma. Part Fibre Toxicol. 2010;7:5.

32. Schulte PA, Kuempel ED, Zumwalde RD, et al. Focused actions to protect carbon nanotube workers. Am J Ind Med. 2012;55:395-411.

33. Hunt G, Riediker M. Building expert consensus on problems of uncertainty and complexity in nanomaterials safety. Nanotechnol Percept. 2011;7:82-98.

34. Organisation for Economic Co-operation and Development. List of Manufactured Nanomaterials and List of Endpoints for Phase One of the Sponsorship Programme for the Testing of Manufactured Nanomaterials: Revision. Paris, France: Organisation for Economic Co-operation and DevelopmentOECD; 2010.

35. Bouwmeester H, Lynch I, Marvin HJP, et al. Minimal analytical characterization of engineered nanomaterials needed for hazard assessment in biological matrices. Nanotoxicology. 2011;5:1-11.

36. Oberdorster G, Oberdorster E, Oberdorster J. Nanotoxicology: an emerging discipline evolving from studies of ultrafine particles. Environ Health Perspect. 2005;113:823-839.

37. Kreyling WG, Semmler M, Erbe F, et al. Translocation of ultrafine insoluble iridium particles from lung epithelium to extrapulmonary organs is size dependent but very low. J Toxicol Environ Health A. 2002;65:15131530 .

38. Oberdorster G, Sharp Z, Atudorei V, et al. Translocation of inhaled ultrafine particles to the brain. Inhal Toxicol. 2004;16:437-445.

39. Oberdorster G, Sharp Z, Atudorei V, et al. Extrapulmonary translocation of ultrafine carbon particles following whole-body inhalation exposure of rats. $J$ Toxicol Environ Health A. 2002;65:1531-1543.

40. Riediker M, Cascio WE, Griggs TR, et al. Particulate matter exposure in cars is associated with cardiovascular effects in healthy, young men. Am J Respir Crit Care Med. 2004;169:934-940.

41. Moller P, Loft S. Oxidative damage to DNA and lipids as biomarkers of exposure to air pollution. Environ Health Perspect. 2010;118:1126-1136.

42. Nel A, Xia T, Madler L, Li N. Toxic potential of materials at the nanolevel. Science. 2006;311:622-627.

43. Poland CA, Duffin R, Kinloch I, et al. Carbon nanotubes introduced into the abdominal cavity of mice show asbestos-like pathogenicity in a pilot study. Nat Nanotechnol. 2008;3:423-428.

44. Takagi A, Hirose A, Nishimura T, et al. Induction of mesothelioma in p53+/mouse by intraperitoneal application of multi-wall carbon nanotube. $J$ Toxicol Sci. 2008;33:105-116.

45. Shvedova AA, Kisin ER, Mercer R, et al. Unusual inflammatory and fibrogenic pulmonary responses to single-walled carbon nanotubes in mice. Am JPhysiol Lung Cell Mol Physiol. 2005;289:L698-L708.

46. Gwinn MR, Vallyathan V. Nanoparticles: health effects-pros and cons. Environ Health Perspect. 2006;114:1818-1825.

47. Brook RD, Rajagopalan S, Pope CA III, et al. Particulate matter air pollution and cardiovascular disease: an update to the scientific statement from the American Heart Association. Circulation. 2010;121:2331-2378.

48. Li N, Nel AE. Feasibility of biomarker studies for engineered nanoparticles: what can be learned from air pollution research. J Occup Environ Med. 2011;53:S74-S79.

49. Suva. Grenzwerte am Arbeitsplatz 2011 [Occupational exposure limits 2011]. Lucerne, Switzerland: Suva; 2011.

50. National Institute for Occupational Safety and Health. Draft Intelligence Bulletin Occupational Exposure to Carbon Nanotubes and Nanofibers. Cincinnati, OH: US Department of Health and Human Services, Centers for Disease Control, National Institute for Occupational Safety and Health; 2010.

51. Antonini JM, Lewis AB, Roberts JR, Whaley DA. Pulmonary effects of welding fumes: review of worker and experimental animal studies. Am J Ind Med. 2003;43:350-360.

52. Liou S-H, Lin M-H, Hsu C-H, et al. The pilot study of specific biomarkers in health effects among engineered nanoparticles manufacturing workers. Epidemiology. 2011;22:S254-S255.
53. Song Y, Li X, Du X. Exposure to nanoparticles is related to pleural effusion, pulmonary fibrosis and granuloma. Eur Respir J. 2009;34:559-567.

54. Erdely A, Hulderman T, Salmen R, et al. Cross-talk between lung and systemic circulation during carbon nanotube respiratory exposure. Potential biomarkers. Nano Lett. 2009;9:36-43.

55. Xia T, Li N, Nel AE. Potential health impact of nanoparticles. Annu Rev Public Health. 2009;30:137-150.

56. Bergamaschi E, Magrini A. Biomonitoring. In: Fadeel B, Pietroiusti A, Shvedova A, eds. Adverse Effects of Engineered Nanomaterials: Exposure, Toxicology, and Impact on Human Health. Amsterdam, the Netherlands: Elsevier; 2012:45-62.

57. Riediker M, Cascio WE, Griggs TR, et al. Health effects in male highway patrol troopers are associated with components of in-vehicle fine particulate matter (PM2.5) attributable to stop-and-go traffic. Am J Respir Crit Care Med. 2004;169:A883.

58. Riediker M, Devlin RB, Griggs TR, et al. Cardiovascular effects in patrol officers are associated with fine particulate matter from brake wear and engine emissions. Part Fibre Toxicol. 2004;1:2.

59. Baccarelli A, Martinelli I, Pegoraro V, et al. Living near major traffic roads and risk of deep vein thrombosis. Circulation. 2009;119:3118-3124.

60. Peters A, von KS, Heier M, et al. Exposure to traffic and the onset of myocardial infarction. N Engl J Med. 2004;351:1721-1730.

61. Lucking AJ, Lundback M, Mills NL, et al. Diesel exhaust inhalation increases thrombus formation in man. Eur Heart J. 2008;29:3043-3051.

62. Leone AM, Gustafsson LE, Francis PL, Persson MG, Wiklund NP, Moncada S. Nitric oxide is present in exhaled breath in humans: direct GC-MS confirmation. Biochem Biophys Res Commun. 1994;201: 883-887.

63. Corradi M, Montuschi P, Donnelly LE, Pesci A, Kharitonov SA, Barnes PJ. Increased nitrosothiols in exhaled breath condensate in inflammatory airway diseases. Am J Respir Crit Care Med. 2001;163:854-858.

64. Bergamaschi E. Occupational exposure to nanomaterials: present knowledge and future development. Nanotoxicology. 2009;3:194-201.

65. Fairchild CI, Stampfer JF. Particle concentration in exhaled breath-summary report. Am Ind Hyg Assoc J. 1987;48:948-949.

66. Simeonova PP, Erdely A. Engineered nanoparticle respiratory exposure and potential risks for cardiovascular toxicity: predictive tests and biomarkers. Inhal Toxicol. 2009;21(suppl 1):68-73.

67. Franco G. Occupational health practice and exposure to nanoparticles: reconciling scientific evidence, ethical aspects, and legal requirements. Arch Environ Occup Health. 2011;66:236-240.

68. Iversen A, Liddell K, Fear N, Hotopf M, Wessely S. Consent, confidentiality, and the Data Protection Act. BMJ. 2006;332:165-169.

69. Hornung RW, Meinhardt TJ. Quantitative risk assessment of lung cancer in US uranium miners. Health Phys. 1987;52:417-430.

70. Stayner L, Smith R, Bailer J, et al. Exposure-response analysis of risk of respiratory disease associated with occupational exposure to chrysotile asbestos. Occup Environ Med. 1997;54:646-652.

71. Nasterlack M, Zober A, Oberlinner C. Considerations on occupational medical surveillance in employees handling nanoparticles. Int Arch Occup Environ Health. 2008;81:721-726.

72. National Institute for Occupational Safety and Health. Interim Guidance for Medical Screening and Hazard Surveillance for Workers Potentially Exposed to Engineered Nanoparticles. DHHS (NIOSH) Publication No. 2009-116. Cincinnati, OH: National Institute for Occupational Safety and Health; 2009.

73. Iavicoli S, Rondinone BM, Boccuni F. Occupational safety and health's role in sustainable, responsible nanotechnology: gaps and needs. Hum Exp Toxicol. 2009;28:433-443.

74. National Institute for Occupational Safety and Health. Exposure assessment and characterization. Approaches to Safe Nanotechnology: Managing the Health and Safety Concerns Associated With Engineered Nanomaterials. Cincinnati, OH: National Institute for Occupational Safety and Health; 2009:23-33.

75. Schulte P, Geraci C, Zumwalde R, Hoover M, Kuempel E. Occupational risk management of engineered nanoparticles. J Occup Environ Hyg. 2008;5:239 249.

76. Gibson RM, Stacey N, Drais E, Wallin H, Zatorski W. Risk Perception and Risk Communication With Regard to Nanomaterials in the Workplace. Luxembourg: European Agency for Safety and Health at Work; 2012. 